\title{
Métodos Para a Avaliação da Usabilidade no Design de Produtos
} Methods for the Usability Evaluation in Product Design

\author{
por Tiago Catecati, Fernanda Gomes Faust, \\ Giorgia Amir Longo Roepke, Fernanda Steinbruch Araujo, \\ Deise Albertazzi, Alejandro Rafael Garcia Ramirez e \\ Marcelo Gitirana Gomes Ferreira
}

\begin{abstract}
RESUMO
Este artigo apresenta uma revisão bibliográfica dos principais métodos de avaliação de usabilidade aplicáveis a produtos industriais. Inicialmente é apresentado o conceito de usabilidade e em seguida, são discutidos os métodos de avaliação de usabilidade de produtos: os que se baseiam nos usuários e os que se baseiam na avaliação de especialistas. Por fim é apresentada uma sistemática para a seleção dos métodos de usabilidade, que está sendo aplicado em um projeto de pesquisa em andamento com foco na avaliação da usabilidade de produtos eletro-eletrônicos com o auxílio da tecnologia de realidade aumentada.
\end{abstract}

Palavras-chave Usabilidade; Avaliação; Desenvolvimento de produto

\section{ABSTRACT}

This paper presents a literature review of the main usability evaluation methods applicable to industrial products. Initially it's presented the concept of usability, applied to industrial products. Then, it's discussed the usability evaluation methods: the ones based on the users of the product and those based on evaluation of usability experts. Also, are presented a systematic for selection of the usability evaluation, that are being applied in an ongoing research project focused on evaluating the usability of electric and electronic products with the help of augmented reality technology.

Keywords Usability; Evaluation; Product Design 


\section{Introdução}

Ao longo das diversas etapas do seu projeto, um produto costuma ser avaliado sob diversos aspectos complementares: função, estética, uso, fabricabilidade, mantenabilidade, impactos sócio-ambientais, entre outros. Tais avaliações se revestem de grande importância, pois subsidiam e impactam as decisões da equipe de projeto. Importa, então, existirem métodos e ferramentas que garantam uma avaliação eficaz do produto sob estes aspectos ao longo do projeto. (BAXTER, 2000; ROZENFELD et al., 2006; BACK et al., 2008).

Este artigo revisa os principais métodos de avaliação de usabilidade de produtos. Primeiramente, se discute o conceito de usabilidade aplicado a produtos tangíveis. Em seguida, se apresentam os seus métodos de avaliação. Dentre os métodos de avaliação que se baseiam em usuários, destacam-se o "pensando em voz alta" e os testes que objetivam a obtenção de dados de natureza quantitativa. Dentre os métodos de avaliação que se baseiam em especialistas, destacam-se o "passo-a-passo cognitivo" e a "avaliação heurística". 0 artigo finaliza discutindo as formas de seleção do método mais adequado para cada produto, considerando os seus usuários e o seu contexto de uso.

\section{Usabilidade}

De acordo com Tullis e Albert (2008), muitas são as definições encontradas na literatura para o termo usabilidade - praticamente uma para cada profissional que trabaIha nesta área, segundo os autores. Estes identificam que, independentemente da definição a ser adotada, sempre se tem: (1) um usuário envolvido; (2) que desenvolve alguma atividade; (3) utilizando um produto, sistema, ou alguma outra coisa. Inicialmente, Krug (2000) - em seu livro clássico "Don't make me think" (p.5) - afirma, de forma bastante simples e direta, que:

Usabilidade significa tão somente garantir que algo funcione bem: que uma pessoa com habilidades e experiências médias (ou mesmo abaixo da média) possam usar a coisa - seja uma página na web, um caça a jato, ou uma porta giratória - para o seu propósito, sem ficar irremediavelmente frustrado.

Numa perspectiva mais voltada para o processo de desenvolvimento de produtos, a Associação de Profissionais em Usabilidade (UPA) declara que:

Usabilidade é uma abordagem para o desenvolvimento de produtos que incorpora, de forma direta, o feedback do usuário ao longo do seu ciclo de desenvolvimento de forma a reduzir custos e a criar produtos e ferramentas que atendam as necessidades dos usuários. 
Por fim, a NBR 9241-11 (p.3), de Agosto de 2002, define usabilidade como: a medida na qual um produto pode ser usado por usuários específicos para alcançar objetivos específicos com eficácia, eficiência e satisfação em um contexto específico de uso.

Para o melhor entendimento desta última definição, cabe esclarecer o significado dos seguintes termos de acordo com Jordan(1998):

- Eficácia: se refere à extensão na qual uma meta é alcançada ou uma tarefa é realizada.

- Eficiência: se refere à quantidade de esforço requerido para se atingir uma meta. Quanto menos esforço, maior é a eficiência.

- Satisfação: se refere ao nível de conforto que os usuários sentem quando utilizam um produto e também ao nível de aceitação do produto pelos usuários para atingir as suas metas.

A definição da NBR 9241-11 deixa claro que a usabilidade não é uma propriedade intrínseca do produto isoladamente. Depende do seu "contexto específico de uso": quem está utilizando o produto; para que finalidade; em qual ambiente - ver Figura 1. Para produtos relacionados ao trabalho, como no caso de um torno mecânico, eficácia e eficiência tendem a ser aspectos mais importantes que satisfação. 0 inverso ocorre com produtos relacionados ao lazer: um jogo eletrônico, por exemplo.

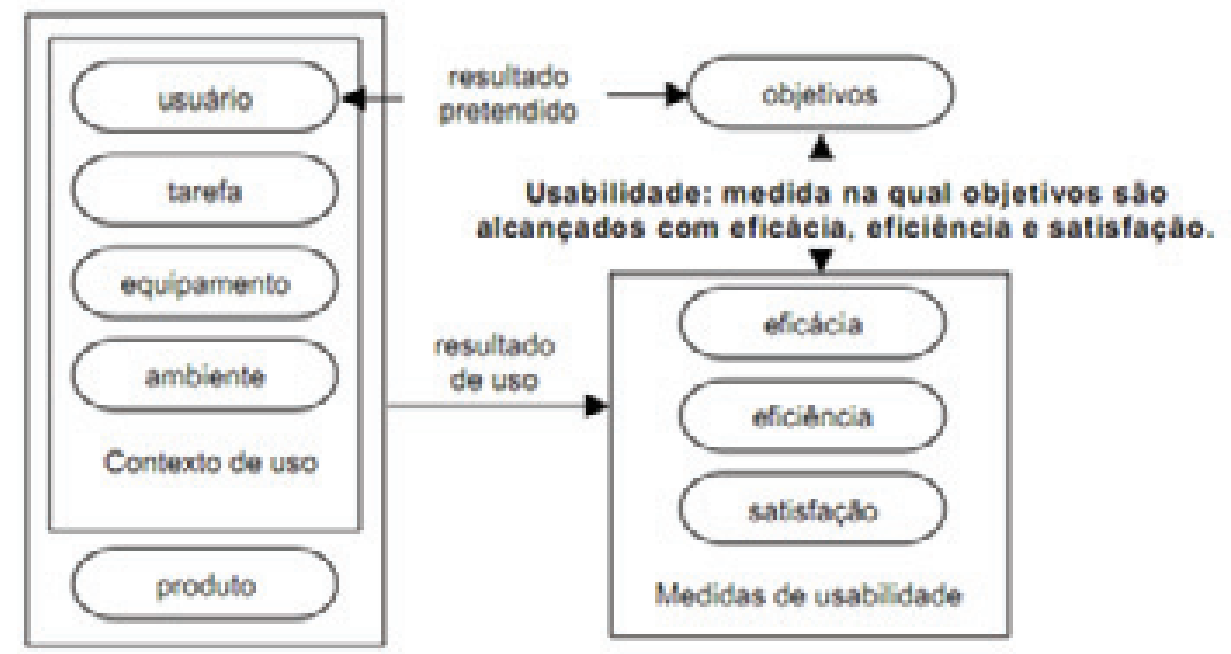

Figura 1. Estrutura de usabilidade. Fonte: NBR 9241-11(2002)

A importância do contexto específico também fica evidenciada quando se examina, por exemplo, a usabilidade de uma torneira automática com sensor infra-vermelho. Bastante encontrada em prédios públicos, demonstra, nestes locais, boa usabilidade: são fáceis de usar e economizam água. Também se mostram bastante higiênicas, visto que os usuários não necessitam tocá-las, minimizando a necessidade de limpeza. A usabilidade desta mesma torneira pode, no entanto, ser questionada se utilizada no contexto de uma residência familiar. 0 seu modo de 
operar não permite o controle de vazão ou temperatura. A experiência de escovar os dentes utilizando este tipo de torneira é, por vezes, bastante desagradável, visto que a aproximação da escova de dente nem sempre é suficiente para acioná-la.

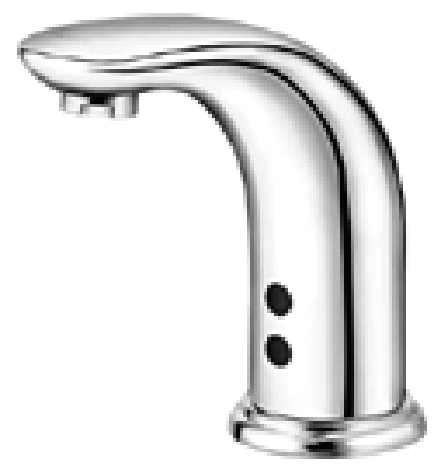

Figura 2. Torneira automática DocolTronic Zenit. Fonte: http://www.docol.com.br

\subsection{Métricas}

De acordo com Jordan (1998), as métricas utilizadas para a avaliação objetiva da usabilidade de um produto, relacionam-se com os três aspectos da usabilidade citados anteriormente.

- Métricas de eficácia: completeza da tarefa; qualidade do resultado.

- Métricas de eficiência: desvios do caminho crítico (o mais eficiente para realizar a tarefa); quantidade ou taxa de erros; tempo para a realização da tarefa; carga mental (medida de diferentes formas).

- Métricas de satisfação: análise qualitativa da satisfação (por meios de entrevistas e questionários, por exemplo); análise quantitativa da satisfação (por meio de escala quantitativa, como a de Likert (LIKERT, 1932) ou do diferencial semântico de Osgood (OSGOOD; SUCl e TANNENBAUM, 1957)

\subsection{Componentes da usabilidade}

Analisando a usabilidade de um produto (ou sistema) - tomando por base a experiência prévia do usuário com o produto - Jordan (1998) propõe cinco componentes nos quais a usabilidade deva ser avaliada.

1. Intuitividade (Guessability): eficácia, eficiência e satisfação com as quais determinados usuários conseguem realizar determinadas tarefas com um produto particular quando o utiliza pela primeira vez.

2. Facilidade de aprendizagem (Learnability): eficácia, eficiência e satisfação com as quais determinados usuários conseguem atingir determinados níveis de desempenho em determinadas tarefas com um produto, tendo já realizado estas tarefas anteriormente. 
3. Desempenho de um usuário experiente: eficácia, eficiência e satisfação com as quais determinados usuários experientes conseguem realizar determinadas tarefas.

4. Potencial do sistema: níveis ótimos de eficácia, eficiência e satisfação com os quais seria possivel realizar determinadas tarefas com um produto.

5. Reusabilidade: eficácia, eficiência e satisfação com as quais determinados usuários conseguem realizar determinadas tarefas depois de tê-las realizada, após um longo período de tempo.

A Figura 3 apresenta uma representação dos cinco componentes da usabilidade.

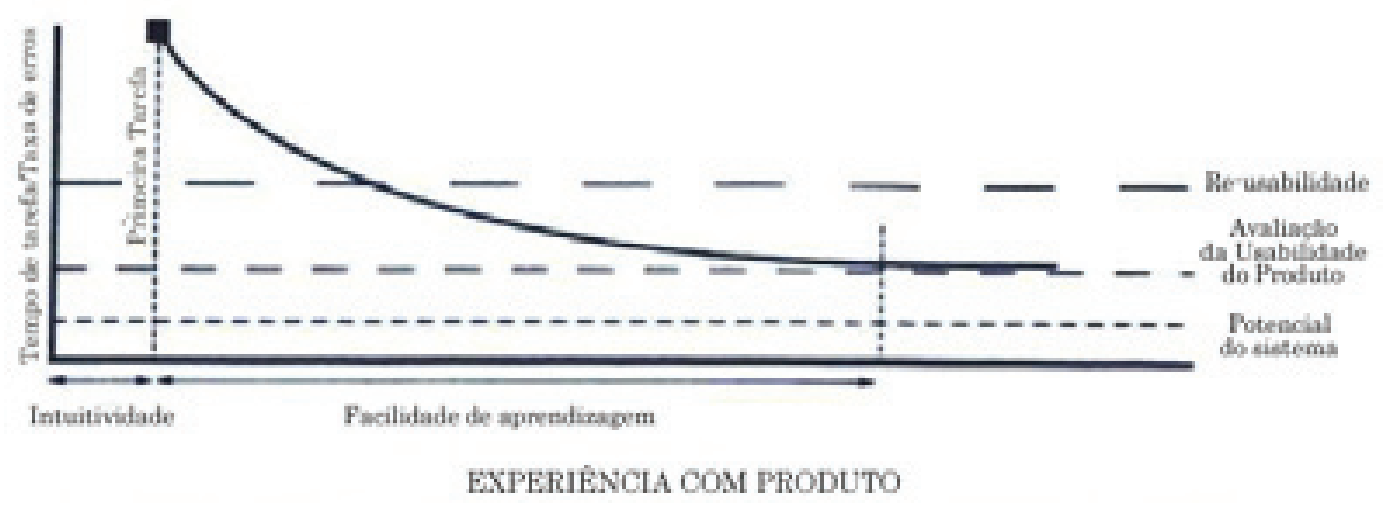

Figura 3. Componentes da usabilidade. Fonte: Jordan, 1998, p.16.

\section{Métodos para a Avaliação da Usabilidade}

Pode-se dividir os métodos para a avaliação da usabilidade de produtos em duas classes: as baseadas em observações e medidas realizadas sobre os usuários e as que se valem do conhecimento de especialistas. Na primeira classe destaca-se 0 Pensando em Voz Alta (Think Aloud Protocol), do qual derivam uma série de outros métodos cujos resultados (métricas) são de natureza essencialmente qualitativa. De forma complementar, provendo resultados quantitativos têm-se as Avaliações de Desempenho. Já na segunda, destacam-se a Avaliação Heurística e o Passo a Passo Cognitivo.

\subsection{Métodos Baseado em Usuários}

Nestes métodos, usuários típicos (reais ou potenciais) realizam tarefas cotidianas com o software, produto ou sistema (ou seus respectivos protótipos) e os avaliadores utilizam os resultados dos testes para analisar como a interface suporta estes usuários na realização das suas tarefas. Para Jordan (1998), não há nada que substitua a possibilidade de ver o usuário tentando utilizar o produto.

3.1.1. Pensando em Voz Alta (e suas Variantes) 
Considerado por Nielsen (1993) o mais valioso dos testes de usabilidade, o método Pensando em Voz Alta (Thinking Aloud Protocol), consiste na narração por parte de usuários das ações realizadas, das decisões tomadas, das suas opiniões e seus sentimentos enquanto interage com o produto (ou protótipo) sob avaliação. De acordo com Jasper (2009), este método consiste em dois estágios: (1) a coleta sistemática das declarações dos usuários; e (2) a análise destas declarações, de forma a se obter um modelo dos processos cognitivos utilizados quando o usuário se defronta com um problema.

Nielsen (1993) salienta que o método pode ser realizado tanto individualmente, quanto em grupo, existindo duas variações: a resposta crítica (critical response) e o relato periódico (periodic report). Na resposta crítica, o usuário reporta as suas ações durante o período de execução da mesma. Já no relato periódico a narrativa e expressão das ações não é possível de ser realizada simultaneamente ao desenvolvimento das ações. Como desvantagem a verbalização da tarefa pode atrasar ou atrapalhar a realização da tarefa.

Diversas variações do método podem ser encontradas na literatura (VORA A HELANDER, 1997; RUBIN, 2008; NIELSEN , 1993), tais como: a aprendizagem por co-descoberta, teste retrospectivo e protocolo de questionamento. A seguir, são apresentadas algumas destas variantes.

\subsubsection{Método Coaching}

Consiste na interação explícita entre o observador e o usuário durante a realização da tarefa. Diferentemente dos outros métodos de avaliação, no método coaching, o usuário pode perguntar ao observador - pessoa que aplica o teste - sobre suas dúvidas relativas ao produto que irá ser testado. Uma variante, envolve um usuário experiente, enquanto o observador analisa a interação do usuário com o produto e a interação entre o usuário e o coach.

De acordo com Nielsen (1993), o propósito desta técnica é descobrir as necessidades dos usuários em termos de informação, proporcionando um melhor treinamento e documentação do produto em desenvolvimento. Também pode ser utilizado no reprojeto da interface do produto, evitando questionamentos por parte dos usuários durante a fase de uso do produto.

\subsubsection{Aprendizagem por Co-descoberta}

Neste método segundo Nielsen (1993) dois usuários exploram juntos um produto e tentam descobrir como realizar determinada tarefa, enquanto são observados. 0 observador analisa as verbalizações dos usuários durante a realização da tarefa, que ocorre de forma mais natural que no método pensando em voz alta.

Como geralmente os usuários testados são amigos ou conhecidos, segundo Dumas at Redish (1999) uma vantagem deste método consiste na informalidade entre os usuários durante a realização da tarefa, pois estes se sentem menos pressionados ao realizar as ações, explorando o problema de forma mais natural. Para Rubin 
(2008) uma desvantagem do método é a possibilidade de distração e desconcentração dos participantes durante as verbalizações do processo, sendo esta também uma desvantagem do método pensando em voz alta.

\subsubsection{Método de Ensino}

Proposto por Vora e Helander (1995) neste método primeiramente são selecionados usuários que irão interagir com o sistema de tal forma que se familiarizem com o mesmo e consigam realizar tarefas pré-determinadas. Após este período de familiarização, para cada usuário "experiente" é introduzido um usuário novato, que desconhece por completo o sistema. Cada usuário experiente é solicitado a apresentar o sistema ao usuário novato e a ensiná-lo a realizar um conjunto pré-determinado de tarefas.

De acordo com Vora e Helander (1995), este método é capaz de gerar um número superior de verbalizações e facilita a captura dos modelos mentais utilizados pelos usuários, na realização das tarefas e na resolução de problemas. Como desvantagem do método proposto, aponta-se um maior tempo necessário para a sua realização.

\subsubsection{Protocolo de Questionamentos}

Este se diferencia do método Pensando em Voz Alta, pois além de deixar que 0 usuário verbalize espontaneamente os seus pensamentos, 0 avaliador estimula 0 usuário realizando perguntas diretas sobre o produto - enquanto realiza tarefas com o produto ou um protótipo. Por meio de perguntas o avaliador busca compreender os modelos mentais dos usuários relativos ao sistema e às tarefas.

\subsubsection{Método Shadowing}

Nesta variante, um usuário experiente com o sistema senta-se ao lado do avaliador e explica o comportamento do usuário que realiza as tarefas. Esta técnica é utilizada quando não é apropriado ou possível que o usuário fale durante a realização das tarefas. A confiabilidade das informações levantadas pode ser reduzida, visto que não são obtidas diretamente do usuário que realiza a tarefa.

\subsubsection{Teste Retrospectivo}

De acordo com Nielsen (1993) o Teste Retrospectivo funciona como uma ferramenta complementar ao método Pensando em Voz Alta ou às suas variantes, podendo ser utilizado conjuntamente. Essa técnica propicia realizar uma análise mais profunda dos acontecimentos sucedidos, possibilitando um maior esclarecimento dos mesmos.

\subsubsection{Teste Remoto}

Esse método, conforme Rex Hartson et.al.(1996), consiste na aplicação do teste de usabilidade onde o observador e o participante estão em diferentes locais ou tempo. Assim, os participantes que estão sendo testados não podem ser observados 
diretamente no processo. Com a maior velocidade da Internet, os aprimoramentos dos recursos e a infinidade de programas de captura de imagem e áudio em tempo real possibilitam a execução de um teste de usabilidade à distância com bom retorno.

\subsubsection{Testes para Medição de Desempenho}

Para Nielsen (1993) e Soken et.al (1993) essa ferramenta é aplicada durante a realização de uma tarefa, possibilitando a medição do desempenho dos usuários, coletando os dados quantitativos dos participantes a partir de parâmetros pré-determinados como porcentagem de sucesso dos testes completados, freqüência e tipo dos erros cometidos, tempo necessário para a realização da tarefa, frustração dos participantes, entre outros. Para garantir uma coleta de dados de forma fiel e precisa, evita-se o contato entre o observador e os participantes do teste, sendo que este deve ser realizado em um laboratório de usabilidade, para minimizar possíveis interferências no processo. É necessária a presença de, no mínimo, cinco participantes na realização deste teste, sendo oito o número ideal para a produção de dados fidedignos.

Nielsen (1993) descreve três passos para a realização de Testes para Medição de Desempenho: (a) definição das metas do teste; (b) condução dos testes; e (c) análise dos dados para a obtenção das conclusões.

\subsubsection{Definição das Metas do Teste}

As metas do teste de medição de desempenho devem primeiramente ser definidas em termos dos atributos de usabilidade a serem investigados: facilidade de aprendizagem, eficiência no uso, facilidade de lembrar, poucos erros, prazer proporcionado, entre outros. Tais atributos devem ser avaliados a fim de obter a importância relativa entre os mesmos. Finalizando a etapa de definição das metas, devem-se buscar medidas que quantifiquem os atributos de usabilidade definidos, tais como:

- Tempo que o usuário realização de uma tarefa específica.

- Número de tarefas (de diversos tipos) que podem ser realizadas em determinado tempo.

- Relação entre interações bem sucedidas e erros.

- Tempo despendido na recuperação de erros.

- Número de comandos ou outros recursos nunca utilizados pelo usuário.

- Número de recursos do sistema no qual o usuário se recorda após uma seção de utilização do produto.

- Freqüência de uso dos manuais e/ou sistema de ajuda e o tempo despendido neste uso.

- Proporção de usuários que dizem preferir usar o sistema em relação a algum sistema similar. 
- Proporção de usuários que utilizam estratégias eficientes de trabalho nos casos em que existem múltiplas formas de realizar as tarefas.

\subsubsection{Condução dos Testes}

Na etapa de condução dos testes, deve-se assegurar que não haverá interrupções, nem fatores que possam perturbar a execução dos testes: ruídos, calor, frio, entre outros. Testes-piloto devem ser realizados para garantir a eficácia das ferramentas e técnicas de coleta de dados. Quando possível, recomenda-se que o teste seja gravado em vídeo. Assim, alguns dados podem ser verificados, ou mesmo coletados, após a realização dos testes, assistindo às gravações de vídeo.

\subsubsection{Análise dos Dados para a Obtenção das Conclusões}

Embora os Testes para Medição de Desempenho objetivem a coleta de dados quantitativos, é importante que durante a execução destes testes se busque coletar dados qualitativos que auxiliem os avaliadores a revelar os processos mentais dos usuários e outras informações que se "escondem" por trás dos dados quantitativos. Tais dados de natureza qualitativa devem ser considerados nas conclusões a serem tiradas pelos avaliadores.

Na etapa final, de análise dos dados para a obtenção das conclusões, podem-se comparar os resultados obtidos com um valor de referência (benchmark), utilizando-se estatística descritiva (medidas centrais e de dispersão). É comum realizar comparações entre os dados obtidos a partir de diferentes projetos (de interface com o usuário), podendo-se utilizar, neste caso, de estatística inferencial (testes de hipótese).

\subsection{Outras Formas de Avaliar a Usabilidade (Baseadas em Usuários)}

Por meio de observações e questionamentos, avaliadores obtêm informações sobre o que agrada ou desagrada o usuário e sobre as suas necessidades. Também podem compreender o sistema conversando com os usuários, observando-os em seu dia-dia ou pedindo que responda a algumas questões, verbalmente ou por escrito.

\subsubsection{Observação em Campo}

Nesta modalidade de avaliação, o especialista vai a campo observar o usuário em seu ambiente natural de trabalho (ou interação), a fim de entender como eles utilizam o sistema (ou produto) para realizar as suas tarefas e compreender os modelos mentais que os mesmos possuem relativos a estes sistemas. Este método pode ser utilizado nas fases de teste e do desenvolvimento do produto.

Nielsen (1993) sugere que a escolha dos usuários a serem observados se dê da maneira mais variada possível, considerando repertório, localização, estilo de vida, entre outros quesitos, com o intuito de captar os diversos panoramas da percepção do produto. 
Uma vantagem proporcionada por essa técnica é que o usuário se encontra em seu ambiente natural, portanto as posturas assumidas por ele serão mais verdadeiras e fiéis do que as interações visualizadas em laboratório. No entanto, as observações de campo acarretam a desvantagem de uma exigência maior do tempo, devido a fatores sob os quais o observador não possui controle, como interrupções e ruídos do sistema.

\subsubsection{Grupos de Foco}

Grupos de Foco é uma técnica que é utilizada para uma avaliação de caráter mais subjetivo, consiste em uma reunião de discussão sobre alguma questão em foco onde podem ser abordadas as experiências de uso, exigências para um novo produto, informações sobre as tarefas realizadas, problemas de usabilidade em relação a um produto, entre outras necessidades avaliadas nos testes (NIELSEN, 1993).

A realização desse tipo de avaliação normalmente acontece na presença de um especialista no assunto que tenha a missão e a habilidade de direcionar o grupo nos debates com um roteiro para determinados tipos de discussões e linhas de raciocínio, mantendo assim o grupo focado.

\subsubsection{Entrevistas}

As entrevistas consistem na aplicação de questões formuladas baseadas nas principais áreas de interesse nas quais as informações necessitam ser levantadas. As entrevistas são realizadas de forma direta, podendo ou não serem estruturadas (NIELSEN, 1993).

Entrevistas realizadas de maneira não estruturada buscam levantar o máximo de informações possível acerca das experiências e expectativas do usuário. Por outro lado, as entrevistas estruturadas apresentam um conjunto pré-determinado de questões que visam guiar e direcionar a entrevista.

\subsubsection{Registro do Uso Real}

Comumente utilizado na avaliação da usabilidade de sistemas computacionais, consiste na coleta automática - e freqüentemente em tempo real - de dados relativos à interação do usuário com o sistema/produto. Especial atenção deve ser dada aos recursos que se relacionam a erros freqüentemente detectados no sistema e também aos recursos relacionados à freqüentes consultas aos itens de ajuda (helps e FAQs) do sistema.

Nielsen (1993) recomenda sempre que possível, a utilização simultânea deste método em combinação com outros métodos em que o usuário pode explicar as suas ações realizadas. 


\subsubsection{Estudo de Campo}

Baseado em Nielsen (1993) esta é uma técnica de grande importância no desenvolvimento do sistema/produto, pois analisa o usuário e suas interações com 0 sistema/produto, observando detalhadamente diversos aspectos que somente são identificados no ambiente e contexto do usuário.

Durante um estudo de campo o investigador observa o usuário em seu contexto, identificando as suas necessidades, fazendo anotações e perguntas aos usuários. Essa observação pode ser realizada de forma direta, quando o observador encontra-se presente durante a realização das tarefas dos usuários, ou indireta, quando a tarefa é acompanhada através de uma gravação de vídeo, por exemplo.

As observações em campo são usualmente utilizadas nas fases iniciais do projeto do produto, a fim de coletar o máximo de dados sobre o usuário e guiar o processo de desenvolvimento do produto. Uma possível desvantagem do método se relaciona com os custos envolvidos para a sua realização: deslocamentos, gravações, entre outros.

\subsubsection{Questionários}

Os questionários são importantes ferramentas de pesquisa que visam à coleta de dados referentes a um grupo representativo da população. Utiliza-se quando se deseja adquirir mais conhecimento a cerca de determinado assunto, de forma simultânea, economizando tempo do pesquisador e facilitando o levantamento estatístico dos dados. Existem três tipos de questionários que podem ser aplicados aos usuários: os fechados, os abertos e os mistos. Todos devem seguir os princípios da clareza e neutralidade.

- Questionários fechados: em cada questão a escolha de resposta se dá dentro de um conjunto de alternativas disponíveis. 0 usuário seleciona a que melhor representa seu ponto de vista.

- Questionários abertos: permitem que o respondente - usuário do produto no caso - elabore suas respostas de acordo com seu ponto de vista e com suas próprias palavras, não precisando se restringir as alternativas pré-definidas pelo pesquisador.

- Questionários mistos: deve ser aplicado quando existe a necessidade de questões tanto abertas quanto fechadas, a fim de proporcionar uma coleta de informações mais completas.

\subsection{Métodos Baseados em Especialistas}

Nesta categoria de métodos, especialistas analisam aspectos relacionados com a usabilidade da interface usuário-produto ou sistema. Dois são os principais métodos de avaliação de usabilidade que se baseiam em especialistas: o Passo a Passo Cognitivo (Cognitive Walkthrough) e a Avaliação Heurística (Heuristic Evaluation). 


\subsubsection{Passo a Passo Cognitivo (e suas Derivações)}

No passo a passo cognitivo um avaliador ou um grupo de avaliadores - especialistas em usabilidade, ergonomia, design, engenharia, marketing, entre outros - inspecionam a interface do sistema/produto com o usuário, realizando um conjunto de testes e avaliando a sua facilidade de entendimento e de aprendizagem. Falhas de projeto são costumeiramente identificadas por meio do passo a passo cognitivo.

A utilização deste método, de acordo com Nielsen (1993), é mais fortemente recomendada nas fases iniciais do processo de design do produto, quando a interface com o usuário pode tomar a forma de mock-up. Nada impede, entretanto, que o método seja utilizado nas fases posteriores. 0 método também pode ser utilizado em um produto já completamente desenvolvido ou mesmo já lançado no mercado. Torna-se uma poderosa ferramenta para a análise de um produto concorrente em um benchmark competitivo.

Devem ser considerados os repertórios e conhecimentos dos usuários que participam do passo a passo cognitivo, assim como a experiência que os mesmos detêm sobre a interface na qual estão trabalhando. Para cada tarefa realizada pelo usuário deve conter uma explicação de como esta deve ser realizada antes do usuário aprender e interpretar a interface.

No passo a passo cognitivo são examinadas todas as ações que o usuário realizou para executar determinadas tarefas, e para isso os avaliadores fazem quatro perguntas durante o teste. A primeira pergunta - será que o usuário conquistou o efeito certo? - diz respeito ao atingimento correto do objetivo, para que desta forma o avaliador analise a interpretação do usuário em cada passo da tarefa. Na segunda pergunta - será que o usuário perceberá que a ação correta está disponível? - trata da compreensão e da visibilidade da ação correta a ser executada pelo usuário. No terceiro inquirimento - será que o usuário associa o fato de ter executado determinada função com o término da tarefa? - diz respeito à correta interpretação e entendimento do usuário a cerca das funções desempenhadas no decorrer da tarefa. Na última pergunta - se a ação correta for executada, será que o usuário perceberá que o progresso está sendo realizado para a solução da tarefa? - onde o avaliador consegue analisar o feedback que está sendo fornecido pelo sistema após cada ação desempenhada pelo usuário.

\subsubsection{Passo a Passo Pluralista}

0 passo a passo pluralista é utilizado nos estágios de avaliação inicial do projeto onde um grupo de usuários, projetistas e engenheiros reúnem-se a fim de discutir e avaliar a usabilidade em um sistema, analisando o cenário e como cada elemento influencia na execução da tarefa (BIAS, 1994).

A grande vantagem desta ferramenta é a de fornecer diversas perspectivas para a avaliação de usabilidade do sistema. Aconselha-se a formação de uma equipe com muitos participantes que apresentem diferentes repertórios e conhecimentos, a fim de que diversos problemas relativos à usabilidade do sistema possam ser 
identificados. Durante o processo é necessário que uma pessoa assuma o papel de facilitador, que deverá esclarecer qualquer dúvida que possa impedir os participantes de completarem a tarefa proposta.

No passo a passo pluralista todos os participantes envolvidos, usuários, projetistas e engenheiros, recebem regras e instruções de como será a sessão e de como proceder na mesma, assim como a descrição da tarefa e do sistema em que se encontram. Durante o processo todos os participantes devem assumir o papel de usuários do sistema em questão. Inicialmente o sistema é apresentado aos usuários, e após, todos escrevem como executariam a tarefa, com a maior riqueza de detalhes possivel. Essa etapa ocorre de maneira individual, sem a interferência de qualquer participante. Após os participantes escreverem os passos necessários para executarem a tarefa, discutem estes mesmos passos e os problemas encontrados no decorrer do processo. Após os usuários terminarem de relatar sua experiência com o sistema, os especialistas relatam suas descobertas ao grupo e depois os projetistas explicam as suas escolhas. Caso o grupo apresente dúvidas em como proceder durante o processo o facilitador explica a todos qual a correta seqüência de etapas necessárias para completar a tarefa em questão. Após todo este processo ocorrer, os participantes respondem um questionário a cerca da usabilidade da interface em questão.

\subsubsection{Avaliação Heurística}

Avaliação Heurística é um principio que orienta uma decisão ou revisão de projeto. Para Nielsen (1993) esse tipo de avaliação consiste em verificar os possíveis problemas de usabilidade. Um conjunto de avaliadores distintos, ergonomistas ou especialistas em usabilidade, que apresentam pouco ou nenhum envolvimento com o projeto, avaliam o sistema. Esse método não necessita da presença de usuários do sistema em questão.

Para a execução dessa técnica é necessária a presença de no mínimo cinco avaliadores, que irão, individualmente, executar a avaliação e ao final discutir e compilar os resultados.

Nielsen (1993) e Jordan (1998) sugerem a utilização de procedimentos heurísticos para auxiliar a avaliação, tais como:

- Simplicidade e naturalidade de diálogo: As informações devem ser apresentadas de forma lógica, clara, natural e objetiva.

- Correspondência entre a linguagem do sistema e a do usuário: As informações contidas no sistema devem apresentar um diálogo claro e familiar, não contendo jargões técnicos do sistema;

- Minimizar a carga de memória do usuário: A interface deve ser coerente e apresentar linguagem unificada fazendo com que a carga da memória do usuário seja minimizada; 
- Consistência: Deve existir uma consistência e padronagem dos dados, prevenindo assim que o usuário faça uma errônea interpretação. Para facilitar o reconhecimento pelo usuário do sistema as opções disponíveis devem apresentar a mesma formatação.

- Feedback: É necessário que o sistema apresente um feedback imediato das ações realizadas, mantendo o usuário informado a cerca do sistema;

- Saídas claramente identificadas: 0 sistema deve fornecer diversas formas de saídas para qualquer possível problema que possa vir acontecer, ou seja, devem ser possibilitadas as opções de desfazer ou refazer determinada ação;

- Atalhos: 0s atalhos do sistema servem para otimizar e acelerar o processo de utilização do mesmo, portanto, usuários experientes ao utilizarem dessa ferramenta, aumentam a velocidade de interação com o sistema.

- Mensagem de erro: As mensagens de erros devem ser expressas de forma clara e objetiva, indicando precisamente qual é o problema e fornecer uma solução construtiva para o mesmo.

- Prevenção de erros: Ao projetar o sistema deve-se atentar a prevenção de possíveis erros que possam vir a acontecer durante a realização de determinadas tarefas.

- Ajuda e documentação: 0 sistema deve fornecer ajuda ao usuário quando necessário, com informações claras e precisas, focando nas tarefas do usuário em questão.

\subsubsection{Inspeção de Características e Funcionalidades}

Essa técnica analisa somente as características e aspectos utilizados para a realização de uma determinada tarefa. Cada característica é avaliada em termos de requisitos de usabilidade, tais como compreensibilidade, viabilidade, eficácia, entre outros.

Essa forma de inspeção consiste basicamente na identificação de todos os aspectos utilizados na execução de uma ação e de como cada um desses desempenha um papel de importância para a realização da tarefa.

\subsubsection{Inspeção baseada em perspectiva}

Devido a dificuldade para detectar todos os problemas referentes à usabilidade do sistema, este método divide entre os inspetores os aspectos de usabilidade em sub-conjuntos e cada um deles organiza sessões a fim de detectar um maior número de problemas no sistema. Dessa forma, cada sessão possui uma perspectiva diferente, coletando assim um maior número de informações necessárias para a otimização do sistema.

Pesquisas mostram que a inspeção baseada em perspectiva detecta cerca de $30 \%$ mais de problemas referentes à usabilidade. Para Zhang, Basili e Shneiderman (1998) 
o objetivo desta técnica consiste em determinar um procedimento mais específico para cada inspetor, direcionando cada vez mais a sessão de inspeção, detectando assim uma porcentagem maior de problemas referentes a esta perspectiva. A combinação de diversas inspeções baseadas em perspectivas resulta na descoberta de um maior número de problemas do que os identificados em outros tipos de inspeções.

\section{Seleção do Método de Usabilidade}

Ao revisar a literatura, percebe-se a inexistência de uma sistemática clara, ou de consenso, a respeito de como selecionar um método de avaliação de usabilidade. No entanto, ao examinar a Figura 1, é possível identificar ao menos dois importantes fatores a serem considerados neste processo: 0 tipo de produto a ser avaliado e o seu contexto de uso.

No que diz respeito ao tipo de produto a ser avaliado, aqueles cuja função prática (ou técnica) predomina - uma furadeira ou um caixa eletrônico, por exemplo tendem a ser mais adequadamente avaliados por meio de métodos que forneçam resultados mais quantitativos, tais como os testes e experimentos com o usuário. Já os produtos que levam a uma maior experiência de uso - aparelhos celulares ou mesmo automóveis, por exemplo - são usualmente avaliados por métodos mais subjetivos: entrevistas, focus group, entre outros.

0 contexto de uso diz respeito ao usuário, à tarefa, ao equipamento e ao ambiente em que o produto será utilizado. Todos esses fatores influenciam na seleção do método de avaliação da usabilidade. Neste sentido, uma mesma bicicleta sendo utilizada a trabalho por um carteiro deve ser avaliada diferentemente de quando utilizada para passeios a lazer.

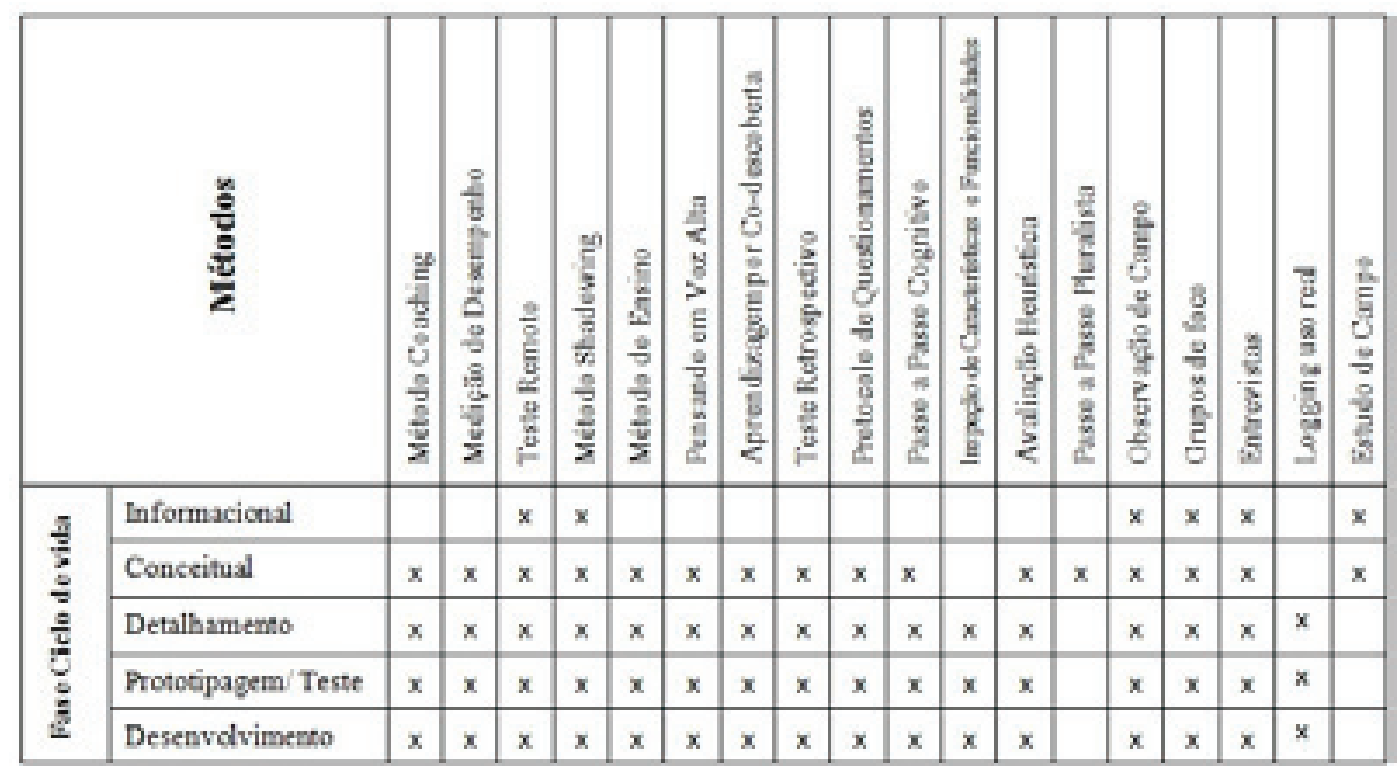

Figura 4. Seleção de Métodos. Fonte: Os autores. 
Outro aspecto a ser considerado é a fase de desenvolvimento que o produto se encontra. Como ilustrada na figura 4. Por exemplo, na fase conceitual os métodos baseados em usuário e em especialistas podem ser utilizados, diferente do informacional que é baseado principalmente em especialistas.

Entre os fatores que podem influenciar o processo de escolha de um método, pode-se considerar também o tempo necessário para realizar um método, o custo de realização, a confiança na obtenção de dados, entre outros quesitos (KARAT, 1994).

\section{Conclusão}

Diante do aumento crescente da complexidade dos produtos e da diversidade de usuários - com diferentes conhecimentos e habilidades - que têm acesso aos produtos, observou-se uma maior necessidade e importância do estudo da usabilidade no desenvolvimento dos produtos. Na revisão bibliográfica, pode-se perceber que apesar do constante aumento de pesquisadores na área, a maioria das pesquisas baseiam-se em Jacob Nielsen. Outra questão observada foi em relação aos métodos de avaliação de usabilidade, onde a maioria são direcionados para softwares e web, havendo uma escassez de métodos para avaliação de produtos.

A presente pesquisa contribui para a sistematização do conhecimento para o nosso idioma, onde existem poucas referências para pesquisa voltada para a aplicação de metodologia no desenvolvimento de produtos e também para a seleção do método de avaliação a ser aplicado em tais produtos. A escolha do método mais apropriado para a avaliação permite que se obtenham resultados mais satisfatórios e de acordo com os objetivos da avaliação. Esta seleção depende do produto, do contexto de uso, do ambiente no qual será utilizado, assim como depende de questões como tempo, custo e o conhecimento dos especialistas. A pesquisa também contribui para a aplicação prática do conhecimento (ou competência, de uma forma mais ampla) no aperfeiçoamento dos produtos (principalmente eletrônicos, por normalmente terem maior complexidade de uso). Produtos projetados levando-se em conta aspectos ergonômicos de usabilidade têm maiores possibilidades de sucesso no mercado. Melhores produtos geram empresas mais competitivas que, por sua vez, geram empregos e renda. 
Referências Bibliográficas

> ASSOCIAÇÃO BRASILEIRA DE NORMAS TÉCNICAS. NBR 9241-11: requisitos ergonômicos para trabalho de escritórios com computadores: parte 11 - orientações sobre usabilidade. Rio de Janeiro, p.3, 2002.

$>$ BACK, N. ET al. Projeto integrado de produtos: planejamento, concepção e modelagem. Manole, (2008).

> BAXTER, M. Projeto de produto. Edgard Blucher. São Paulo, 2000.

> BIAS, R. G. The pluralistic usability walkthrough: coordinated empathies. In Nielsen, J. and Mack, R. (eds.),Usability inspection methods, John Wiley a Sons, New York, 63-76. 1994.

> CYBIS, Walter, BETIOL, Adriana H., FAUST, Richard. Ergonomia e usabilidade. São Paulo: Novatec, 2007.

$>$ DUMAS, Joseph S.; REDISH, Janice C. A practical guide to usability testing. Intellect, 1999.

> JASPER, Monique W.M. A comparison of usability methods for testing interactive health technologies: Methodological aspects and empirical evidence. International journal of medical informatics. v. 78, n. 5, p. 340-353, 2009.

$>$ HARTSON Rex H. et. al., Remote Evaluation: The Network as an Extension of the Usability Laboratory, in CHI96 Conference Proceedings, 1996.

> HELANDER, Martin G., LAUNDAUER, Thomas K., e PRABHU, Prasad V. (editors). Handbook of

> Human-Computer Interaction. Amsterdam. Elsevier Science B. V. 1997.

$>$ JORDAN, Patrick W. What is usability? In: ROBERTSON, S. (Ed.) Contemporary ergonomics. London: Taylor at Francis, 1994. pp. 454-458.

> JORDAN, Patrick W. An introduction to usability. London: Taylor at Francis, 1998.

$>$ KARAT, C. M. A comparison of user interface evaluation methods. Ed. John Wiley ct Sons, Inc, p. 203-233, 1994.

> KRUG, Steve. Don't make me think: a common sense approach to web usability. New Riders, 2000, pp.5.

> LIKERT, Rensis. A technique for the measurement of attitudes. Archives of psychology. 140. 1932. pp. 1-55.

> NIELSEN, Jakob. Usability engineering. San Diego (CA): Academic Press, 1993.

> NIELSEN, Jakob; MACK, Robert L. Usability inspection methods. New York: John Willey at Sons, 1994.

> NORMAN, Donald. La psicologia de los objetos cotidianos. Madrid: Nerea, 1988.

> OSGOOD, Charles E., SUCl, George J., TANNENBAUM, Percy H. The measurement of meaning. Urbana: The University of Illinois, 1957. 
> ROZENFELD, H. et al. Gestão de desenvolvimento de produtos: uma referência para a melhoria do processo. São Paulo: Saraiva, 2006.

> RUBIN, Jeffrey; CHISNEL, Dana. Handbook of usability testing: how to plan, design and conduct effective tests. 2nd ed. Indianápolis: John Wiley đa Sons, 2008.

> SOKEN et al, 1993, N.Soken, Reinhart, B., Vora, P., and Metz, S. (1993), Methods for Evaluating Usability (Section $5 \mathrm{~B}$ ), Honeywell.

> USABILITY PROFESSIONAL'S ASSOCIATION. What's usability? Disponível em: <http:// www.upassoc.org/usability_resources/about_usability/index.html>. Acesso em: 11 de maio de 2011.

> VORA, P.; HELANDER, M. A teaching method as an alternative to the concurrent think-aloud method for usablity testing. In: ANZAI, Y.; OGAWA, K.; MORI, H. (Editors) Symbiosis of human and artifact: human and social aspects of human-computer interaction. Amsterdam: Elsevier, 1995. pp.375-380.

> TULLIS, Thomas; ALBERT, Willian. Measuring the user experience: collecting, analyzing, and presenting usability metrics. Burlington: Morgan Kaufman, 2008.

> ZHANG Z.; BASILI V.; SCHNEIDERMAN B. An empirical study of perspective-based usability inspection. Proceedings of the Proceedings of the

> Human Factors and Ergonomics Society 42nd Annual Meeting, Chicago, 1998.

Tiago Catecati, Universidade do Estado de Santa Catarina - Bolsista

tiagocatecati@hotmail.com

Fernanda Gomes Faust, Universidade do Estado de Santa Catarina

fernandagfaust@gmail.com

Giorgia Amir Longo Roepke, Universidade do Estado de Santa Catarina - Bolsista giorgiaroepke@hotmail.com

Fernanda Steinbruch Araujo, Universidade Federal do Estado de Santa Catarina - Doutoranda em Engenharia de Produção

feujo@hotmail.com

Deise Albertazzi, Universidade Federal do Paraná - Mestranda do Design da UFPR

dalbertazzig@gmail.com

Prof. Alejandro Rafael Garcia Ramirez, Universidade do Estado de Santa Catarina - Professor Visitante

garcia.ramirez@gmail.com

Prof. Dr. Marcelo Gitirana Gomes Ferreira, Universidade do Estado de Santa Catarina - Coordenador da bolsa I Professor

marcelo.gitirana@gmail.com 